\title{
GMR
}

\section{Correlation between interleukin- 6 expression in articular cartilage bone and osteoarthritis}

\author{
X.Q. Qu ${ }^{1 *}$, W.J. Wang ${ }^{2 *}$, S.S. Tang ${ }^{3}$, Y. Liu ${ }^{1}$ and J.L. Wang ${ }^{1}$ \\ ${ }^{1}$ Orthopaedic Institute of PLA, 89th Hospital, Weifang, China \\ ${ }^{2}$ Department of Nephrology PLA, 89th Hospital, Weifang, China \\ ${ }^{3}$ Department of Joint Surgery, the Affiliated Hospital of Weifang Medical University, \\ Weifang, China \\ *These authors contributed equally to this study. \\ Corresponding author: J.L. Wang \\ E-mail: JianLiWang2014@126.com
}

Genet. Mol. Res. 14 (4): 14189-14195 (2015)

Received February 20, 2015

Accepted June 25, 2015

Published November 13, 2015

DOI http://dx.doi.org/10.4238/2015.November.13.2

\begin{abstract}
This study aimed to investigate the expressional profile of interleukin-6 (IL-6) in articular cartilage bone of osteoarthritis (OA) patients and its correlation with OA. A total of 30 articular cartilage bone samples from knee OA patients, which were collected by knee arthroscopy or articular surgery, comprised the study group, and 30 samples of normal articular cartilage tissue comprised the control group. Both mRNA (messenger ribonucleic acid) and protein levels of IL-6 and matrix metalloproteinase- 9 (MMP-9) were measured and compared, and a correlation analysis was performed between the two. The integral optical density (IOD) values of MMP-9 and IL-6 proteins in the study group were $9.21 \pm 3.22$ and $8.94 \pm$ 3.17 , respectively; these were significantly higher $(P<0.05)$ than those in the control group at $3.14 \pm 1.48$ and $6.64 \pm 1.53$, respectively. The IOD values of mRNA transcripts for MMP-9 and IL-6 in the study group were $8.31 \pm 2.28$ and $8.78 \pm 3.43$, respectively; these were significantly higher than the values in the control group at $3.52 \pm 1.37$ and $5.21 \pm 1.72(P<$ $0.05)$, respectively. Further, the correlation analysis revealed significantly positive relationships for both protein $(r=0.434, P=0.001)$ and $\operatorname{mRNA}(r=$
\end{abstract}


0.413, $\mathrm{P}=0.002$ ) levels between MMP-9 and IL-6. In conclusion, articular cartilage tissues in knee OA patients have higher levels of MMP-9 and IL-6 expression, and these may play a synergistic role in OA pathogenesis.

Key words: Osteoarthritis; Articular cartilage bone; Interleukin-6; Correlation analysis

\section{INTRODUCTION}

Osteoarthritis (OA), a common orthopedic condition, primarily presents as chronic, advancing, and degenerative pathological changes in osteoarticular tissues characterized by degradation and decrease of articular cartilage matrix, formation of osteophytes, and aseptic synovial inflammation, leading to arthralgia and joint dysfunction (Burguera et al., 2014). Detailed information on pathogenesis of $\mathrm{OA}$ is not available yet, although the inflammatory lesion of joint tissues in OA is similar to that seen in rheumatoid arthritis (Koskinen et al., 2014). Some studies revealed increased activity of inflammatory factor interleukin-6 (IL-6) and matrix metalloproteinase-9 (MMP-9) produced by inflammatory synovial tissues, activated chondrocytes, and infiltrative inflammatory cells (Wang et al., 2006). As an important cytokine participating in the degradation of articular cartilage, IL-6 has pluripotent roles in immunomodulation, regulation of cell growth and differentiation, and induction of inflammation (Ai et al., 2014). Over-secretion of the MMP family can induce excess degradation of extracellular matrix of the articular cartilage, causing degenerative changes such as erosion, ulceration and absence/disappearance of articular cartilage (Wada et al., 2014). This study measured expression levels of IL-6 and MMP-9 in cartilage tissues from 30 OA patients and analyzed the correlation between IL-6 and MMP-9 in an attempt to elucidate a new concept in the study of OA pathogenesis.

\section{MATERIAL AND METHODS}

\section{Patient characteristics}

The protocol of this study was approved by the PLA, 89th Hospital (Shandong, China). Informed consent was obtained from all subjects. Articular cartilage bone tissue samples obtained during arthroscopic knee surgery or articular surgery from 30 knee OA patients admitted to our hospital between February 2011 and April 2014 comprised the study group, and 30 samples of normal articular cartilage bone tissues comprised the control group. The study group included 17 women and 13 men (age range, 39-72 years; average age, 54.2 \pm 8.6 years). The control group consisted of 19 women and 11 men (age range, 33-70 years; average age, $52.3 \pm 9.2$ years). There were no significant differences in gender or age between the study and control groups $(P>0.05)$.

\section{Diagnostic criteria}

Knee OA was diagnosed based on the following 1986 American Rheumatism Association guidelines (Sakao et al., 2008): 1) prolonged knee pain in the past month; 2) crepitus during knee movement; 3) morning stiffness lasting <30 min; 4) knee tissue swelling with snapping sounds from joint; 5) knee tissue swelling without snapping sounds; and 6) serological test. Knee OA diagnosis 
was made on the basis of occurrence of a combination of characteristics (1) + (2) + (3) + (4) or $(1)+(2)+(3)+(5)$ or $(1)+(6)$ and X-ray examinations using the Kellgren-Lawrence classification grades. All patients in the control group were diagnosed with a simple meniscus injury.

\section{Sample collection and process}

Articular cartilage tissues were collected by joint debridement under an arthroscope or during knee arthroplasty in OA patients, whereas samples for the control group were obtained during surgery for simple meniscus injury. As previously described (Li et al., 2013), all cartilage samples were first fixed in $4 \%$ paraformaldehyde for $24 \mathrm{~h}$, followed by gradient dehydration ( $100 \%$ ethanol for $30 \mathrm{~min}, 2$ times; $95 \%$ ethanol for $30 \mathrm{~min}$; $85 \%$ ethanol for $30 \mathrm{~min}$; $75 \%$ ethanol for $30 \mathrm{~min}$; and xylene for $10 \mathrm{~min}, 2$ times) and paraffin embedding, and were sectioned into $4-\mu \mathrm{m}$ thick slices by a microtome (Leica, Germany). Tissue slices were then mounted onto glass slides, which were dried at $62^{\circ} \mathrm{C}$, and were re-hydrated by sequential immersion in xylene $(5$ min, 2 times), $100 \%$ ethanol (5 min), 95\% ethanol (5 min), 80\% ethanol ( $5 \mathrm{~min}), 75 \%$ ethanol (5 min), and distilled water (2 min). Protein levels of IL-6 and MMP-9 were then assayed using the immuohistochemical (IHC) method.

\section{Detection of mRNA on paraffin-based tissue slices utilizing in situ hybridization (ISH) technique}

In brief, tissue slices were re-hydrated as described in IHC staining, followed by $0.01 \%$ Triton X-100 immersion for $2 \mathrm{~min}$, proteinase $\mathrm{K}(0.25 \mathrm{~g} / \mathrm{L})$ digestion for $5 \mathrm{~min}$ at room temperature, and blocking in $2 \mathrm{~g} / \mathrm{L}$ histamine for $2 \mathrm{~min}$. Tissue slices were then progressively dehydrated, denatured at $80^{\circ} \mathrm{C}$ for $2 \mathrm{~min}$, and frozen on ice. Pre-hybridization was performed for $3-4 \mathrm{~h}$ at $42^{\circ} \mathrm{C}$ in a humidified chamber with a pre-hybridization buffer. After washing away the pre-hybridization buffer, IL-6 or MMP-9 probes with pre-denaturation were added onto slices for hybridization at $42^{\circ} \mathrm{C}$ overnight. On the next day, tissue slices were washed using $2 \mathrm{X}$ SSC buffer, $0.1 \%$ Triton $\mathrm{X}-100$ for $2 \mathrm{~min}$, and buffer 1 ( $1 \mathrm{M} \mathrm{NaCl}, 0.1 \mathrm{M}$ Tris, $\mathrm{pH} 7.5)$ and were blocked in buffer 2 (3 g bovine serum albumin in $100 \mathrm{~mL}$ buffer 1) for $1 \mathrm{~h}$. After washing away buffer 2, anti-biotin conjugated with alkaline phosphatase was added, incubated at $42^{\circ} \mathrm{C}$ for $20 \mathrm{~min}$, and washed by buffer 1 . The tissue slice was developed using nitro-blue tetrazolium chloride chromogenic substrate in dark for $30 \mathrm{~min}$, covered with coverslips, and observed under a microscope. All procedures followed the manual instructions of the ISH kits for IL-6 (Boshi BioTech Corp., China) and MMP-9 (Maixin Bio. Corp., China).

\section{Index for microscopic observation}

The intracellular brown granule in microscopic images indicated positive expression of MMP-9 or IL-6 protein, whereas intracellular blue-purple granules represented positive signals for MMP-9 or IL-6 mRNAs. The integral optical density (IOD) value on IHC and ISH staining images was measured and analyzed by the CMIAS medical image analysis system (China). In brief, one representative positive cell was marked in each one of five randomly selected high-magnification (200X) fields, and all positive cells in other fields were automatically detected. Averaged IOD values represented the density of protein/mRNA granules. 


\section{Statistical analysis}

All collected data were entered in Excel 2003 and analyzed by SPSS ver.17.0 package (IBM Corp., USA). All data with normal distributions are reported as mean \pm SEM. The independent 2-sample Student $t$-test was used for comparisons of means, and Pearson's correlation coefficient was used for correlative analysis. The significance level was defined as $\alpha=0.05$.

\section{RESULTS}

\section{Protein and mRNA expression levels of MMP-9 and IL-6}

In the study group, the IOD values of MMP-9 and IL-6 protein were $9.21 \pm 3.22$ and $8.94 \pm$ 3.17, respectively, which were significantly higher than $3.14 \pm 1.48$ and $6.64 \pm 1.53$ in the controls $(P<0.05)$. The IOD values of mRNA for MMP-9 and IL-6 in the study group were $8.31 \pm 2.28$ and $8.78 \pm 3.43$, respectively, which were also significantly higher than $3.52 \pm 1.37$ and $5.21 \pm 1.72$ in the controls $(P<0.05)$, as listed in Table 1. Representative IHC images of IL-6 and MMP-9 in articular cartilage of knee OA patients are shown in Figure 1.

\begin{tabular}{|c|c|c|c|}
\hline Group & $\mathrm{N}$ & MMP-9 (IOD) & IL-6 (IOD) \\
\hline \multicolumn{4}{|l|}{ Protein } \\
\hline Study group & 30 & $9.21 \pm 3.22$ & $8.94 \pm 3.17$ \\
\hline Control group & 30 & $3.14 \pm 1.48$ & $6.64 \pm 1.53$ \\
\hline t value & & 9.382 & 3.579 \\
\hline$P$ value & & 0.000 & 0.000 \\
\hline \multicolumn{4}{|l|}{ mRNA } \\
\hline Study group & 30 & $8.31 \pm 2.28$ & $8.78 \pm 3.43$ \\
\hline Control group & 30 & $3.52 \pm 1.37$ & $5.21 \pm 1.72$ \\
\hline t value & & 9.863 & 5.096 \\
\hline$P$ value & & $<0.000$ & $<0.000$ \\
\hline
\end{tabular}

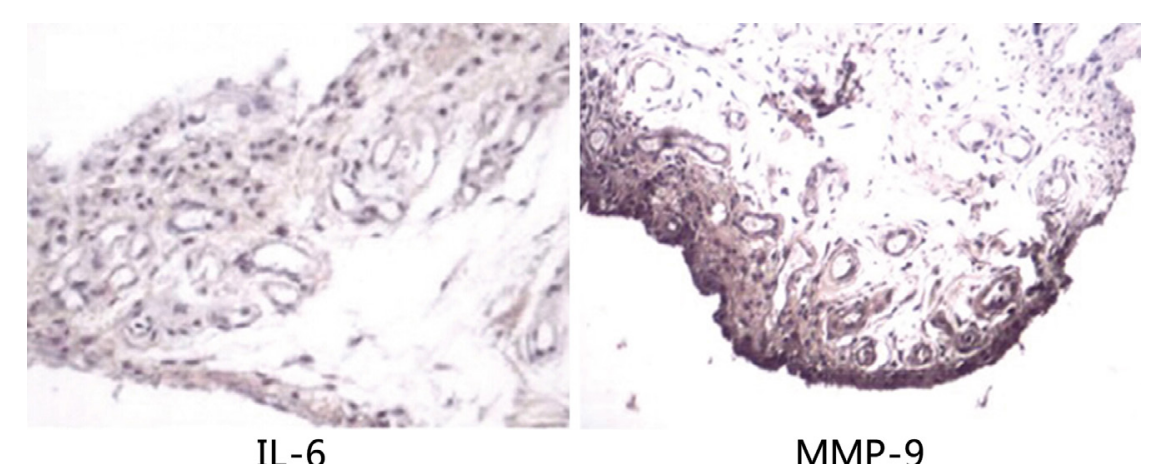

IL-6

MMP-9

Figure 1. IHC staining images (200X) showing positive signals of IL-6 (left) and MMP-9 (right) proteins.

\section{Correlation analysis between MMP-9 and IL-6 levels in knee OA patients}

A correlation analysis between MMP-9 and IL-6 in 30 knee OA patients showed significantly 
positive relationships between the protein $(r=0.434, P=0.001)$ and mRNA levels $(r=0.413, P=$ 0.002 ) of these two factors. Scatter diagrams with regression lines are presented in Figure 2.
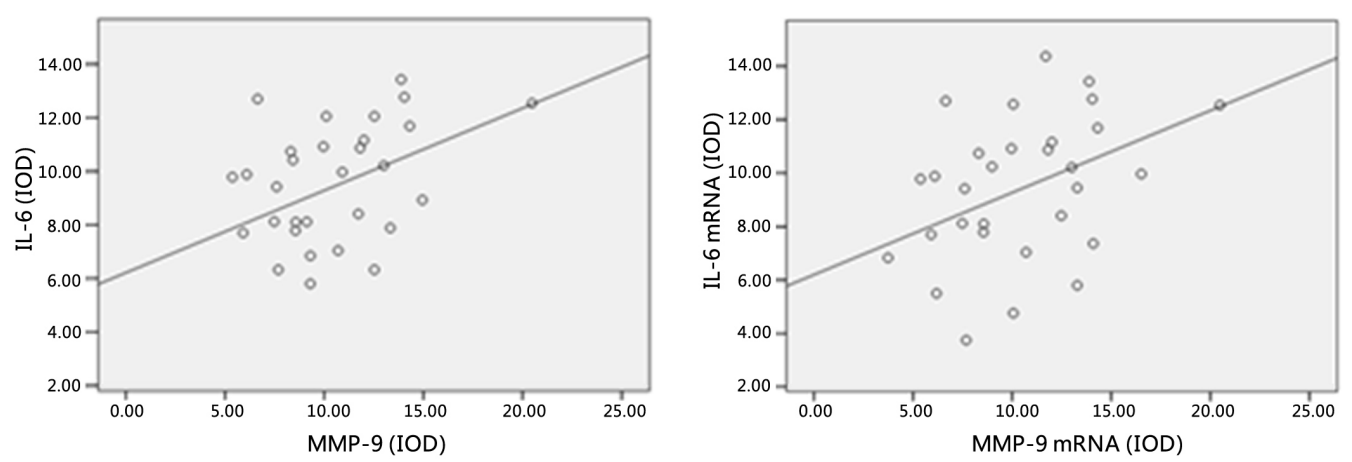

Figure 2. Scatter diagrams of protein (left) and mRNA (right) levels between MMP-9 and IL-6.

\section{DISCUSSION}

OA, a disease of articular cartilage and subchondral bone, is induced by systemic or locally produced cytokines and involves an advancing destructive dysfunction of articular cartilage in a series of processes including repair and reconstruction of articular cartilage and osteophyte formation on the edges (Zanotti and Canalis, 2013). During progression of the disease, articular synovial inflammation produces cytokines including IL-1, IL-6, and TNF- $\alpha$, which induce secretion of a cartilage-degrading proteinase such as MMP by chondrocytes, thus advancing the degenerative changes in the joints (Ahn et al., 2013). Although some aspects of OA pathogenesis are known, its detailed mechanism still remains unclear. This study measured expression levels of IL-6 and MMP9 in OA patients' cartilage bones and revealed significantly higher protein and mRNA levels of IL-6 and MMP-9 in patients compared to controls, suggesting the participation of IL-6 and MMP-9 in the pathological changes of OA.

Located in the short arm of chromosome 7, IL-6 gene codes for a protein consist of 212 amino acid residues. IL- 6 is mainly secreted by mono-macrophages, vessel endothelial cells, and fibroblasts, and can activate B cells and $T$ cells to participate in pathophysiological processes including immunomodulation, cell growth and differentiation, and the inflammatory response (Lee et al., 2012). In OA, IL-6 can stimulate chondrocytes and synovial cells to produce prostaglandin, collagenase, and metalloproteinase, which induces cartilage degradation. IL-6 can be secreted by chondrocytes in both normal individuals and OA patients, and plays an important role in the proliferation and metabolism of cartilage (Grassel et al., 2010). This study showed an elevated expression of IL- 6 in the bones of OA patients, possibly due to the reason that IL- 6 secretion from monocytes was facilitated by inflammatory factors including IL-1 $\beta$ and prostaglandin $E$ as a consequence of the inflammatory response in the synovial tissues in $\mathrm{OA}$; this suggests that elevated IL-6 and synovial inflammation are probably caused by damage of articular cartilage, which normally functions as a major secretion site for IL-6 (Lipari and Gerbino, 2013).

As a member of the MMP family, MMP-9 is a $\mathrm{Zn}^{2+} / \mathrm{Ca}^{2+}$-dependent proteinase and works as an important enzyme that induces metabolism of the cellular matrix (Guo et al., 2014). MMP. 
9 is produced by OA cartilage osteoblasts as well as activated osteoclasts and increases the absorptive capacity of osteoclasts via digestion of bone matrix, thereby causing destruction of bone (Li et al., 2012a). Articular cartilage is a connective tissue consisting of $99 \%$ cartilage matrix and $1 \%$ chondrocytes, with no blood vessels, lymph, or nerve tissues (Li et al., 2012b). Under physiological conditions, a dynamic balance is maintained between degradation and synthesis of cartilage matrix under the direction of MMPs and TIMPs (tissue inhibitor of metalloproteinases). After the damage of articular cartilage, however, high levels of MMPs are secreted by articular chondrocytes and synovial cells, leading to erosion, ulceration, and disappearance of articular cartilage (Guo et al., 2011). This study showed significantly higher than normal levels of protein and mRNA expression of MMP-9 in OA patients' bones, suggesting further destruction of articular cartilage by activated MMP-9 in OA.

This study also performed a correlation analysis between IL-6 and MMP-9 levels in the bones of OA patients, in whom IL-6 level was positively related with MMP-9 with respect to protein and mRNA, which were also correlated with the status of the cartilage disease, suggesting a possible interaction between IL-6 and MMP-9 in the pathogenesis of OA. Therefore, the articular cartilage damage in OA is caused by a synergistic effect of MMP-9 and cytokine IL-6. A possible mechanism is the elevated MMP-9 secretion induced by higher IL-6 expression from monocytes, which is activated by the synovial inflammatory response, thereby disturbing the balance between MMPs and TIMPs and leading to the damage of chondrocytes (Guo et al., 2011). In this process, IL-6 stimulates the production of prostaglandin and collagenase in normal cartilage and synovial cells in order to stimulate the IL-1 induced-MMP response in synovial fibroblasts in rheumatoid arthritis (Litinsky et al., 2006; Wang et al., 2010; Shen et al., 2011) or to elevate the expression of VEGF (vascular endothelial growth factor) type II receptor, thus facilitating MMP-9 secretion (Sakao et al., 2008; Kim et al., 2011).

In summary, IL-6 and MMP-9 participate in cartilage damage in OA, which induces synovial inflammation and high expression levels of IL-6. Elevated IL-6 further stimulates cartilage and synovial cells to secrete PEG and collagenase, facilitating MMP-9 production and sensitivity of IL-1 in response to MMP-9, thereby degrading cartilage and further worsening the damage to articular cartilage. Therefore, the interaction between IL-6 and MMP-9 plays an important role in the occurrence of OA and illustrates a new concept in the pathogenesis of OA.

\section{Conflicts of interest}

The authors declare no conflict of interest.

\section{ACKNOWLEDGMENTS}

We thank the anonymous reviewers for reviewing this manuscript.

\section{REFERENCES}

Ahn JK, Huang B, Bae EK, Park EJ, et al. (2013). The role of alpha-defensin-1 and related signal transduction mechanisms in the production of IL-6, IL-8 and MMPs in rheumatoid fibroblast-like synoviocytes. Rheumatology 52: 1368-1376.

Ai Z, Ning X, Shou T, Tang W, et al. (2014). Association of interleukin-6 promoter polymorphism with knee osteoarthritis: a meta-analysis. Chin. Med. J. 127: 2492-2496.

Burguera EF, Vela-Anero A, Magalhaes J, Meijide-Failde R, et al. (2014). Effect of hydrogen sulfide sources on inflammation and catabolic markers on interleukin 1beta-stimulated human articular chondrocytes. Osteoarthritis Cartilage 22: 1026-1035. 
Grassel S, Beckmann J, Rath B, Vogel M, et al. (2010). Expression profile of matrix metalloproteinase-2 and -9 and their endogenous tissue inhibitors in osteonecrotic femoral heads. Int. J. Mol. Med. 26: 127-133.

Guo J, Zhang W, Li Q, Gan H, et al. (2011). Significance of expressions of matrix metalloproteinase 9 mRNA, transforming growth factor beta1, mRNA and corresponding proteins in osteoarthritis. Zhongguo Xiu Fu Chong Jian Wai Ke Za Zhi 25: 992-997.

Guo SL, Han CT, Jung JL, Chen WJ, et al. (2014). Cystatin C in cerebrospinal fluid is upregulated in elderly patients with chronic osteoarthritis pain and modulated through matrix metalloproteinase 9-specific pathway. Clin. J. Pain 30: 331-339.

Kim KS, Choi HM, Lee YA, Choi IA, et al. (2011). Expression levels and association of gelatinases MMP-2 and MMP-9 and collagenases MMP-1 and MMP-13 with VEGF in synovial fluid of patients with arthritis. Rheumatol. Int. 31: 543-547.

Koskinen A, Vuolteenaho K, Moilanen T and Moilanen E (2014). Resistin as a factor in osteoarthritis: synovial fluid resistin concentrations correlate positively with interleukin 6 and matrix metalloproteinases MMP-1 and MMP-3. Scand. J. Rheumatol. 43: 249-253.

Lee YA, Choi HM, Lee SH, Yang HI, et al. (2012). Synergy between adiponectin and interleukin-1beta on the expression of interleukin-6, interleukin-8, and cyclooxygenase-2 in fibroblast-like synoviocytes. Exp. Mol. Med. 44: 440-447.

Li H, Li L, Min J, Yang H, et al. (2012a). Levels of metalloproteinase (MMP-3, MMP-9), NF-kappaB ligand (RANKL), and nitric oxide (NO) in peripheral blood of osteoarthritis (OA) patients. Clin. Lab. 58: 755-762.

Li H, Miao SB, Dong LH, Shu YN, et al. (2012b). Clinicopathological correlation of Kruppel-like factor 5 and matrix metalloproteinase-9 expression and cartilage degeneration in human osteoarthritis. Pathol. Res. Pract. 208: 9-14.

Li Y, Frank EH, Wang Y, Chubinskaya S, et al. (2013). Moderate dynamic compression inhibits pro-catabolic response of cartilage to mechanical injury, tumor necrosis factor-alpha and interleukin-6, but accentuates degradation above a strain threshold. Osteoarthritis Cartilage 21: 1933-1941.

Lipari L and Gerbino A (2013). Expression of gelatinases (MMP-2, MMP-9) in human articular cartilage. Int. J. Immunopathol. Pharmacol. 26: 817-823.

Litinsky I, Paran D, Levartovsky D, Wigler I, et al. (2006). The effects of leflunomide on clinical parameters and serum levels of IL-6, IL-10, MMP-1 and MMP-3 in patients with resistant rheumatoid arthritis. Cytokine 33: 106-110.

Sakao K, Takahashi KA, Mazda O, Arai Y, et al. (2008). Enhanced expression of interleukin-6, matrix metalloproteinase-13, and receptor activator of NF-kappaB ligand in cells derived from osteoarthritic subchondral bone. J. Orthop. Sci. 13: 202-210.

Shen PC, Wu CL, Jou IM, Lee CH, et al. (2011). T helper cells promote disease progression of osteoarthritis by inducing macrophage inflammatory protein-1gamma. Osteoarthritis Cartilage 19: 728-736.

Wada TT, Araki Y, Sato K, Aizaki Y, et al. (2014). Aberrant histone acetylation contributes to elevated interleukin-6 production in rheumatoid arthritis synovial fibroblasts. Biochem. Biophys. Res. Commun. 444: 682-686.

Wang CT, Lin YT, Chiang BL, Lin YH, et al. (2006). High molecular weight hyaluronic acid down-regulates the gene expression of osteoarthritis-associated cytokines and enzymes in fibroblast-like synoviocytes from patients with early osteoarthritis. Osteoarthritis Cartilage 14: 1237-1247.

Wang GW, Wang MQ, Wang XJ, Yu SB, et al. (2010). Changes in the expression of MMP-3, MMP-9, TIMP-1 and aggrecan in the condylar cartilage of rats induced by experimentally created disordered occlusion. Arch. Oral Biol. 55: 887-895.

Zanotti S and Canalis E (2013). Interleukin 6 mediates selected effects of Notch in chondrocytes. Osteoarthritis Cartilage 21: 1766-1773. 